In N nanostructures

\section{InN Nanocrystals, Nanowires, and Nanotubes}

\author{
Kripasindhu Sardar, F. L. Deepak, A. Govindaraj, \\ M. M. Seikh, and C. N. R. Rao*
}

Indium nitride has attracted increasing attention in the last few years because of its properties of potential value in optoelectronic devices and other technologies. ${ }^{[1]}$ There have been several efforts to prepare $\mathrm{InN}$ nanostructures, but the results reported are not altogether complete and satisfactory. Nanocrystals of $\mathrm{InN}$ have been produced by the reaction of $\mathrm{InCl}_{3}$ and $\mathrm{Li}_{3} \mathrm{~N}$ at $250^{\circ} \mathrm{C}$, but the material obtained was a mixture of the cubic and hexagonal phases. ${ }^{[2]}$ The reaction of $\mathrm{In}_{2} \mathrm{~S}_{3}$ and $\mathrm{NaNH}_{2}$ yielded hexagonal nanocrystals with diameters in the range of $10-30 \mathrm{~nm}$, with an absorption band centered around $1.9 \mathrm{eV}^{[3]}$ Indium halide CVD on a $\mathrm{Si}(100)$ substrate in the presence of $\mathrm{NH}_{3}$ has been used to produce flowerlike hexagonal crystals, but the size of the crystals is in the micrometer regime. ${ }^{[4]}$ In the decomposition of azido precursors, a solution-liquid-solid (SLS) process gave rise to $\approx 20 \mathrm{~nm}$ diameter InN fibers which are polycrystalline. ${ }^{[5]}$ Decomposition of another azido precursor yielded InN whiskers of 10-200 nm diameter by the vapor-liquid-solid (VLS) process, but the optical properties of the whiskers have not been reported. ${ }^{[6]}$ InN nanowires have been grown on a gold-coated silicon substrate by the thermal evaporation of pure In metal in the presence of $\mathrm{NH}_{3}$. The nanowires had a diameter in the $40-80 \mathrm{~nm}$ range, with a broad emission peak at $1.85 \mathrm{eV}^{[7]} \mathrm{By}$ employing a VLS route, in which a mixture of $\mathrm{In}_{2} \mathrm{O}_{3}$ and $\mathrm{In}$ metal was reacted with $\mathrm{NH}_{3}$ at $\approx 700^{\circ} \mathrm{C}$, nanowires with diameters in the $10-100 \mathrm{~nm}$ range were obtained, but the optical spectra are not reported. ${ }^{[8]}$ CVD with a mixture of $\mathrm{In}_{2} \mathrm{O}_{3}$ and In along with $\mathrm{NH}_{3}$ on a $\mathrm{Si} / \mathrm{SiO}_{2}$ substrate coated with $\mathrm{Au}(20 \mathrm{~nm}$ thick), gave nanowires of $15-30 \mathrm{~nm}$ diameter with an absorption band at $\approx 1.85 \mathrm{eV}^{[9]}$ We notice that some of the procedures used to produce the InN nanostructures employ relatively high temperatures, which can give rise to defects or cause decomposition. Where low-temperature reactions have been employed, the optical properties reported are not in tune with the present understanding of the material. While most of the papers on $\mathrm{InN}$ nanostructures report the $1.9 \mathrm{eV}$ absorption band as being characteristic of the material, it is now believed that the band characteristic of $\mathrm{InN}$ is in the near-

[*] K. Sardar, F. L. Deepak, Dr. A. Govindaraj, M. M. Seikh, Prof. Dr. C. N. R. Rao Chemistry and Physics of Materials Unit

Jawaharlal Nehru Centre for Advanced Scientific Research Jakkur P.O., Bangalore 560064 (India)

Fax: $(+91) 80-2362-2760$

E-mail: cnrrao@jncasr.ac.in

K. Sardar, Dr. A. Govindaraj, M. M. Seikh, Prof. Dr. C. N. R. Rao Solid State and Structural Chemistry Unit Indian Institute of Science

Bangalore 560012 (India)
IR region. ${ }^{[10-12]}$ Because of the importance of $\mathrm{InN}$ as an electronic material, we considered it important to carry out a systematic investigation of the nanostructures produced at relatively low temperatures, with specific interest in the optical properties. In this communication, we report a study of the nanocrystals, nanowires, and nanotubes of InN prepared by new chemical routes. We also demonstrate that a band around $0.7 \mathrm{eV}$ in the near-infrared region is truly characteristic of InN.

A typical TEM image of the InN nanocrystals obtained by the solvothermal reaction of tris $(N$-nitroso-phenylhydroxylaminato)indium, $\left[\operatorname{In}\left(\mathrm{C}_{6} \mathrm{H}_{5} \mathrm{~N}_{2} \mathrm{O}_{2}\right)_{3}\right]$, and $1,1,1,3,3,3-$ hexamethyldisilazane (HMDS) in toluene at $265^{\circ} \mathrm{C}$ is shown in Figure $1 \mathrm{a}$. The image reveals nanocrystals with an aver-

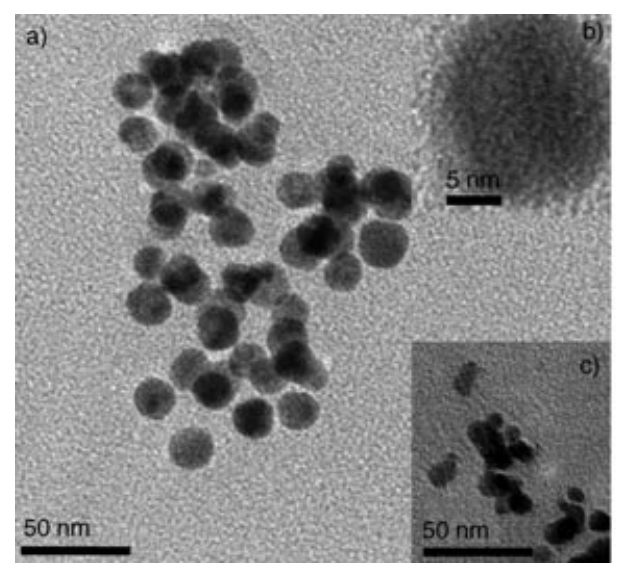

Figure 1. a) TEM image of InN nanocrystals prepared by the reaction of $\left[\ln \left(\mathrm{C}_{6} \mathrm{H}_{5} \mathrm{~N}_{2} \mathrm{O}_{2}\right)_{3}\right]$ and HMDS; b) HREM image of one such nanocrystal; c) nanocrystals prepared by the reaction of $\mathrm{InCl}_{3}$ and HMDS.

age diameter $\approx 15 \mathrm{~nm}$. The nanocrystals are single-crystalline in nature, as evidenced from the high resolution electron microscope (HREM) image (Figure 1b). The lattice fringes correspond to the (101) plane of hexagonal $\mathrm{InN}$ with $d=2.70 \AA$ A. The reaction of $\mathrm{InCl}_{3}$ with $\mathrm{HMDS}$ at $300^{\circ} \mathrm{C}$ also gave nanocrystals (diameter $10-15 \mathrm{~nm}$; Figure $1 \mathrm{c}$ ). The XRD pattern of the nanocrystals (Figure $2 \mathrm{a}$ ) shows characteristic reflections of the wurtzite structure $(a=3.56 \AA, c=$ $5.70 \AA$, JCPDS no.: 02-1450). The average particle size obtained from the Scherrer formula was $\approx 12 \mathrm{~nm}$. The Raman spectra of the nanocrystals gave the characteristic bands due to the $A_{1}(\mathrm{LO}), \mathrm{E}_{2}$, and $\mathrm{A}_{1}(\mathrm{TO})$ modes. ${ }^{[3,13]}$ In Figure $3 \mathrm{a}$ we show the optical absorption spectrum of the InN nanocrystals, which reveals a broad featureless absorption centered around $950 \mathrm{~nm}$ and a well-defined absorption band at $\approx 1575 \mathrm{~nm}(0.79 \mathrm{eV})$. The observation of the latter band in the near-infrared region is significant.

Reaction of indium acetate with HMDS in the absence of a solvent at $300^{\circ} \mathrm{C}$ yielded interesting nanostructures consisting of both nanowires and nanotubes with yields of 40 and $60 \%$, respectively. The XRD pattern of these nanostructures was characteristic of wurtzite InN (Figure $2 b$ ). The SEM image in Figure 4 a shows the presence of a large number of nanowires and nanotubes. The nanowires have diameters in the 50-100 $\mathrm{nm}$ range with lengths extending up to $1 \mu \mathrm{m}$, as seen in the low-resolution TEM images in Fig- 


\section{communications}

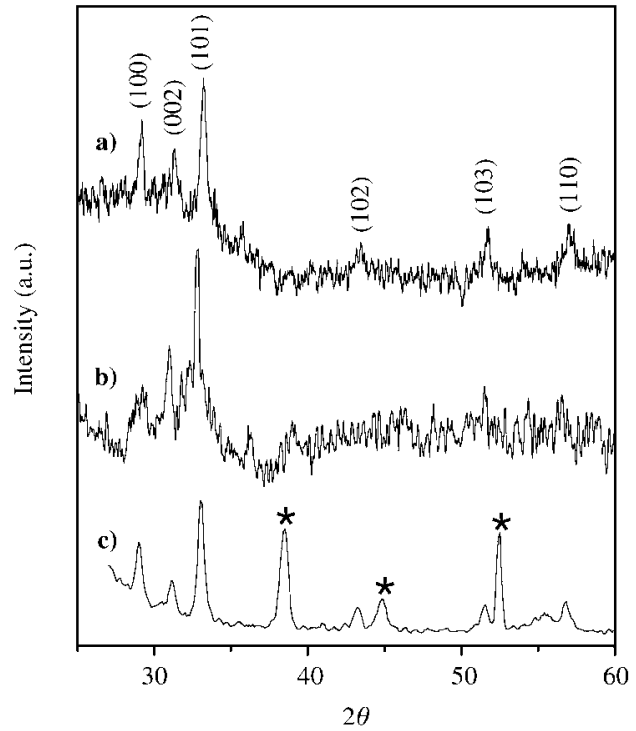

Figure 2. Powder XRD patterns: a) InN nanocrystals obtained from the reaction of $\left[\ln \left(\mathrm{C}_{6} \mathrm{H}_{5} \mathrm{~N}_{2} \mathrm{O}_{2}\right)_{3}\right]$ and HMDS; b) a mixture of nanotubes and nanowires; c) InN nanowires obtained by NSP (grazing angle). Asterisks indicate peaks due to the $\mathrm{Si}(100)$ substrate.

ure $4 \mathrm{~b}$. We have also found the presence of a few Y-junction nanowires (Figure $4 \mathrm{c}$ ). The nanowires are single-crystalline with Bragg spots corresponding to the (100), (101), and (002) planes in the selected-area electron diffraction (SAED) pattern (see inset of Figure 4c). HREM images of the nanowires gave interplanar spacings of $\approx 2.7 \AA$, which corresponds to the (101) plane of the wurtzite structure, with the growth direction of the nanowires being parallel to the (101) plane. Figure 4d and e shows TEM images of the InN nanotubes. They show an interlayer spacing of $3.08 \AA$ corresponding to the (100) planes as shown in the HREM image in Figure $4 \mathrm{f}$. This spacing is distinctly lower than the interlayer spacing of $3.4 \AA$ in carbon nanotubes. The inset in Figure $4 \mathrm{f}$ shows the SAED pattern of the $\mathrm{InN}$ nanotube

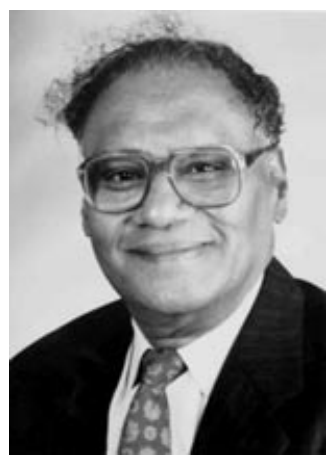

\section{Editorial Advisory Board Member}

C.N.R. Rao obtained his PhD from Purdue University and DSc from Mysore University. He is the Linus Pauling Research Professor and Honorary President of the J. Nehru Centre for Advanced Scientific Research and Honorary Professor at the Indian Institute of Science (both Bangalore). His research interests are mainly in solid-state and materials chemistry (e.g., transition-metal oxides, open-framework structures, and nanomaterials). He has authored nearly 1000 research papers and edited or written 30 books. A member of several academies including the Royal Society, US National Academy of Sciences, and the Pontifical Academy of Sciences, he is currently Distinguished Visiting Professor at the University of California, Santa Barbara. He recently received the Somiya Award of the International Union of Materials Research Societies (IUMRS) and has also been awarded the Einstein Gold Medal of UNESCO and Hughes Medal of the Royal Society.

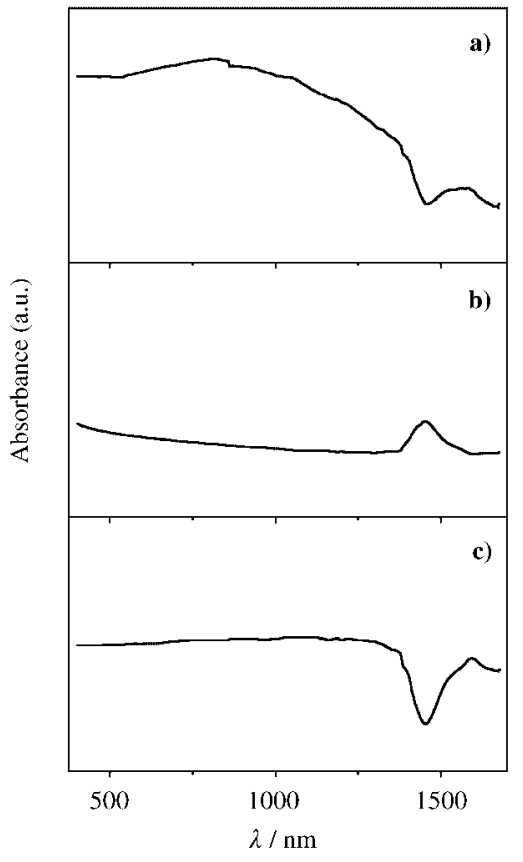

Figure 3. Electronic absorption spectra: a) InN nanocrystals; b) a mixture of InN nanowires and nanotubes (prepared by the reaction of indium acetate with HMDS); c) InN nanowires (prepared on a Si (100) substrate).

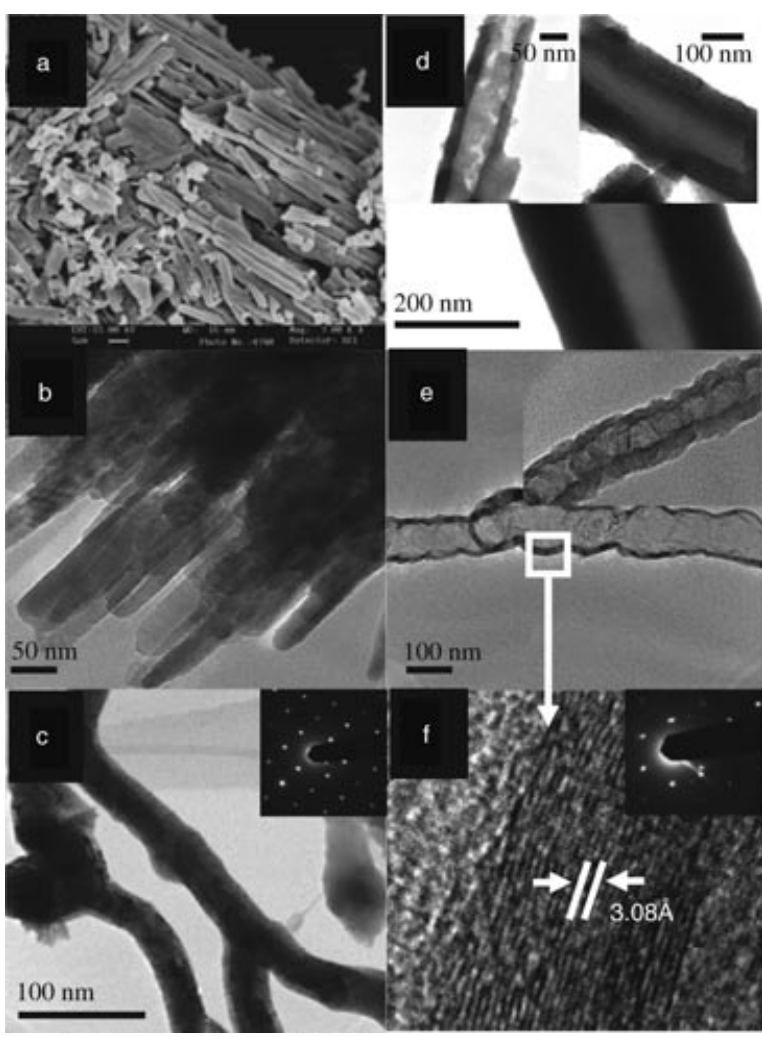

Figure 4. a) SEM image of a mixture of $\operatorname{InN}$ nanowires and nanotubes; b,c) TEM images of nanowires, d,e) TEM images of nanotubes; f) HREM image of a wall of the nanotube. The insets in (c) and (f) show the associated electron diffraction patterns. 
with Bragg spots corresponding to the (100) planes. Optical absorption spectra of the nanowires and nanotubes gave a sharp band around $1475 \mathrm{~nm}(\approx 0.8 \mathrm{eV}$; Figure $3 \mathrm{~b})$. Interestingly, no distinct absorption features were found in the 600 $1000 \mathrm{~nm}$ region.

We have also prepared single-crystalline InN nanowires by the reaction of indium acetylacetonate and $\mathrm{NH}_{3}$ on $\mathrm{Si}(100)$ substrates covered with catalytic gold particles by employing the nebulized spray pyrolysis (NSP) technique. ${ }^{[14]}$ An SEM image (Figure 5a) reveals the high yield of nano-

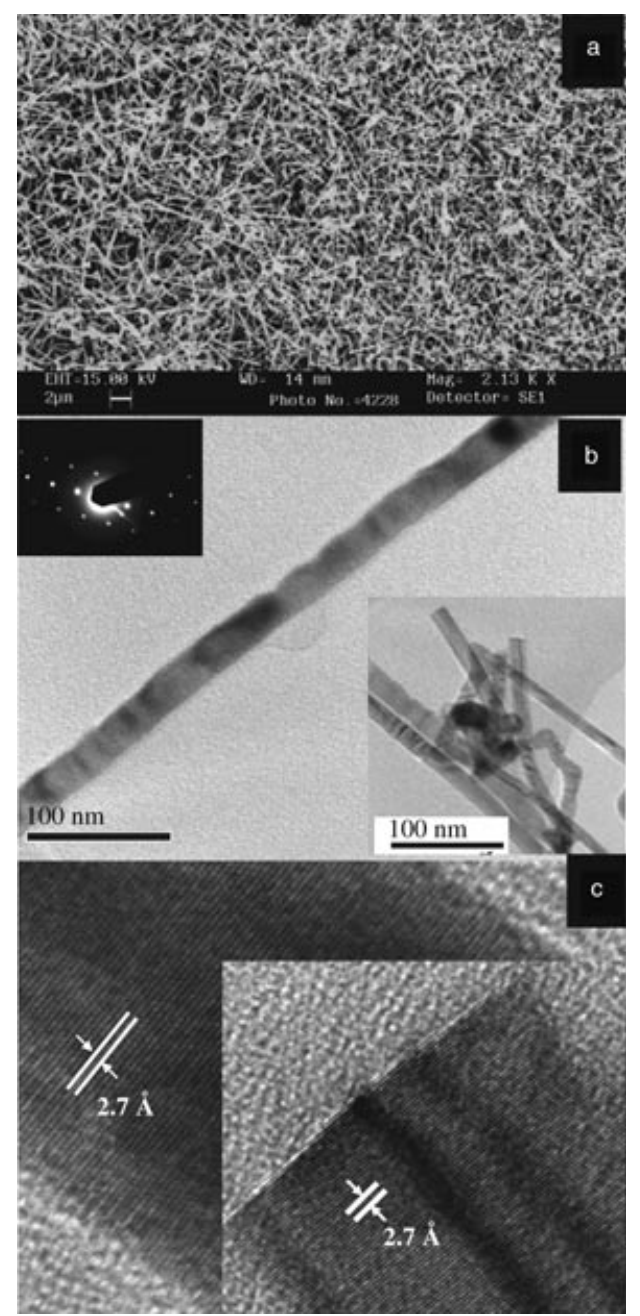

Figure 5. a) SEM image of the InN nanowires obtained by NSP; $b, c)$ TEM and HREM images of the nanowires. The inset in (b) shows the SAED pattern and the inset in (c) shows the HREM image at the tip of the nanowire.

wires that are obtained. The grazing incidence XRD pattern of the nanowires (Figure 2c) is characteristic of the hexagonal structure, and the Raman spectrum is also characteristic of the wurtzite structure. TEM images (Figure $5 \mathrm{~b}$ ) show the nanowires to have diameters in the range of $10-40 \mathrm{~nm}$, with lengths up to a few micrometers. The nanowires are singlecrystalline, as proved by the SAED pattern (inset in Figure $5 \mathrm{~b}$ ), with spots corresponding to the (100), (002), and (101) planes. The HREM images in Figure $5 \mathrm{c}$ show lattice fringes with a spacing of $\approx 2.70 \AA$ corresponding to the (101) planes of the wurtzite structure. The growth of the nanowires is in the $\langle 101\rangle$ direction. The HREM image of the tip of a nanowire shown as an inset in Figure $5 \mathrm{c}$, does not contain any catalyst particle. The optical absorption spectrum of the InN nanowires (Figure $3 \mathrm{c}$ ) shows a broad band in the $\approx 900-1200 \mathrm{~nm}$ region, and a sharp feature around $1575 \mathrm{~nm}(0.79 \mathrm{eV})$.

The prepared InN nanocrystals, nanotubes, and nanowires all show a characteristic sharp band in the near-IR region $(0.7-0.8 \mathrm{eV})$. This is significant since the nanocrystals and nanotubes were prepared at a low temperature (260$300^{\circ} \mathrm{C}$ ), well below the decomposition temperature of $\mathrm{InN}$ $\left(\approx 500^{\circ} \mathrm{C}\right) .{ }^{[15]}$ The nanowires prepared at $\approx 585^{\circ} \mathrm{C}$ also show the $0.8 \mathrm{eV}$ band. We, therefore, propose that the $0.7 \mathrm{eV}$ band is not due to an impurity, such as through the inclusion of metallic In. ${ }^{[10]} \mathrm{Wu}$ et al. ${ }^{[11 \mathrm{a}]}$ have pointed out that the intrinsic bandgap in the $0.7-0.8 \mathrm{eV}$ range is characteristic of InN rather than the commonly reported $1.9 \mathrm{eV}$. The variation of the bandgap between 0.7 and $1.7 \mathrm{eV}$ is reported to arise as a result of the dependence on the electron concentration. ${ }^{[11 \mathrm{a}-\mathrm{b}]}$ Theoretical calculations show that the intrinsic bandgap of $\mathrm{InN}$ is $\approx 0.65 \mathrm{eV}^{[12]}$ The $1.9 \mathrm{eV}$ band has been considered to arise from oxygen incorporation. ${ }^{[16]}$ The absorption spectra reported in the present study clearly show that the band in the $0.7-0.8 \mathrm{eV}$ region is exhibited by all the nanostructures of InN. Because of the absence of a clear band centered around $1.9 \mathrm{eV}$ in the samples prepared by us at low temperatures, we have reason to believe that the $1.9 \mathrm{eV}$ band may not be intrinsic, unlike the $0.7 \mathrm{eV}$ band. Room-temperature PL emission spectra of all the InN nanostructures presented herein gave a band centered at around $675 \mathrm{~nm}$ (Figure 6), but we could not record the low-energy band in the near-infrared region. The $675 \mathrm{~nm}$ PL band arising from defects is known to be characteristic of InN. ${ }^{[7,9]}$ The infrared spectra of the nanostructures showed a band around $450 \mathrm{~cm}^{-1}$ due to In-N stretching mode. ${ }^{[3]}$

In conclusion, InN nanocrystals, nanowires, and nanotubes have been successfully prepared by employing new

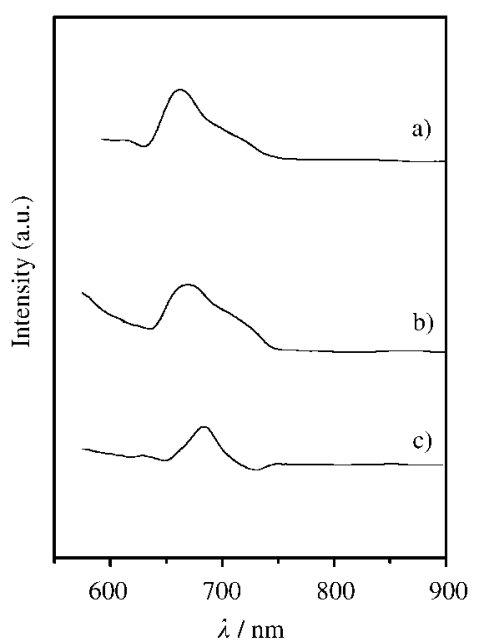

Figure 6. Photoluminescence spectra: a) InN nanocrystals; b) a mixture of InN nanowires and nanotubes; c) InN nanowires. 
chemical routes. The nanostructures have been characterized by X-ray diffraction, electron microscopy, and various spectroscopic methods. It has been shown that the near-infrared band around $0.7 \mathrm{eV}$ is characteristic of these materials.

\section{Experimental Section}

Indium cupferron $\left(\left[\ln \left(\mathrm{C}_{6} \mathrm{H}_{5} \mathrm{~N}_{2} \mathrm{O}_{2}\right)_{3}\right]\right)$ was prepared by the reaction of anhydrous $\mathrm{InCl}_{3}$ (99.999\%, Aldrich) and the ammonium salt of $\mathrm{N}$-nitroso- $\mathrm{N}$-phenylhydroxylamine $\left(\mathrm{C}_{6} \mathrm{H}_{5} \mathrm{~N}(\mathrm{NO}) \mathrm{ONH}_{4}, 97 \%\right.$, Aldrich) in a $1: 3$ molar ratio in deionized water at $0^{\circ} \mathrm{C}$. An instant precipitation was observed. The white precipitate was washed thoroughly with cold deionized water and then dried at room temperature. The white powder obtained was recrystallized from methanol and characterized by thermogravimetric analysis and infrared spectroscopy. $\left[\ln \left(\mathrm{C}_{6} \mathrm{H}_{5} \mathrm{~N}_{2} \mathrm{O}_{2}\right)_{3}\right](0.2 \mathrm{mmol})$ was dissolved in anhydrous toluene $(10 \mathrm{~mL})$. To the solution, 1,1,1,3,3,3-hexamethyldisilazane (HMDS; $5 \mathrm{~mL}, 99.9 \%$, Aldrich) and $\mathrm{N}$-cetyl- $\mathrm{N}, \mathrm{N}, \mathrm{N}$-trimethylammonium bromide (CTAB; $0.3 \mathrm{mmol}$ ) was added. The mixture was stirred for $30 \mathrm{~min}$ and transferred to a stainless steel autoclave $(25 \mathrm{~mL}$ capacity). The autoclave was kept inside an oven maintained at $265^{\circ} \mathrm{C}$. The reaction was carried out for 2-4 days to enhance the crystallinity of the material, although $\operatorname{InN}$ formation was complete within 6$12 \mathrm{~h}$. The black-colored suspension obtained was centrifuged and the precipitate cleaned with absolute ethanol, and dispersed in toluene for further characterization.

To prepare $\mathrm{InN}$ nanocrystals starting from $\mathrm{InCl}_{3}$, anhydrous $\mathrm{InCl}_{3}$ (0.23 mmole, $99.999 \%$, Aldrich) was placed in the stainless steel autoclave $(25 \mathrm{~mL}$ capacity) containing toluene $(10 \mathrm{~mL})$ and CTAB (0.15 mmole). To this solution, HMDS $(5 \mathrm{~mL})$ was added drop by drop and the mixture sonicated for $15 \mathrm{~min}$. The autoclave was placed inside an oven maintained at $300^{\circ} \mathrm{C}$, and the reaction was carried out for between $6-96 \mathrm{~h}$. $\mathrm{InCl}_{3}$ forms a well-defined complex with HMDS at room temperature, ${ }^{[17]}$ which decomposes under solvothermal conditions to give the $\ln N$ nanocrystals.

One-dimensional nanostructures of InN were obtained by the reaction of $\ln (\mathrm{OAc})_{3}$ (0.5 mmole, $99.99 \%$, Aldrich) and HMDS $(5 \mathrm{~mL})$ in a $25 \mathrm{~mL}$ stainless steel autoclave maintained in a furnace at a temperature of $300^{\circ} \mathrm{C}$ for $48 \mathrm{~h}$. No external solvent or surfactant was added and HMDS itself acted as the solvent. After the reaction was complete, the contents were cooled slowly to room temperature. The product was removed by filtration, washed with absolute ethanol and dried. The aspect ratio of these 1D nanostructures could be controlled by varying the relative reactant concentrations and temperature.

Nebulized spray pyrolysis (NSP) was employed to grow $\ln N$ nanowires by the following procedure: A dilute solution $(\approx$ $1 \mathrm{~mm}$ ) containing indium acetylacetonate in methanol was atomized using a PZT transducer. The atomized vapor was passed over the Au-coated $(\approx 50 \AA)$ n-type $\mathrm{Si}(100)$ substrate maintained at $585^{\circ} \mathrm{C}$ inside a quartz tube furnace in flowing $\mathrm{NH}_{3}$.

The nanostructures were characterized by powder X-ray diffraction (XRD) using $\mathrm{Cu}_{\mathrm{Ka}}$ radiation with a Rich-Seifert 3000-TT diffractometer. Scanning Electron Microscope (SEM) images were obtained with a LEICA S440i microscope. Transmission Electron
Microscopy (TEM) images were recorded with a JEOL JEM 3010 microscope operating at an accelerating voltage of $300 \mathrm{kV}$. Optical spectra were recorded at room temperature. Electronic absorption spectra were recorded using a Perkin-Elmer Lambda 900 UV/VIS/NIR spectrophotometer. The samples were dispersed in absolute ethanol or toluene and recorded in the 2000-200 nm range. Photoluminescence (PL) measurements were carried out with a Perkin-Elmer LS 50B luminescence spectrophotometer with an excitation wavelength of $520 \mathrm{~nm}$. Infrared spectra were recorded with a Bruker FT-IR spectrometer. The Raman measurements were performed in a $90^{\circ}$ geometry using a Jobin Yvon TRIAX 550 triple-grating spectrometer equipped with a cryogenic charge-coupled device camera, using a diodepumped frequency-doubled solid-state Nd:YAG laser of $532 \mathrm{~nm}$ (Model DPSS 532-400, Coherent Inc. USA).

\section{Keywords:}

indium $\cdot$ nanocrystals $\cdot$ nanotubes $\cdot$ nanowires $\cdot$ nitrides

[1] F. A. Ponce, D. P. Bour, Nature 1997, 386, 351-359.

[2] Y.-J. Bai, Z.-G. Liu, X.-G. Xu, D.-L. Cui, X.-P. Hao, X. Feng, Q.-L. Wang, J. Cryst. Growth 2002, 241, 189-192.

[3] J. Xiao, Y. Xie, R. Tang, W. Luo, Inorg. Chem. 2003, 42, 107111.

[4] N. Takahashi, A. Niwa, H. Sugiura, T. Nakamura, Chem. Commun. 2003, 318-319.

[5] S. D. Dingman, N. P. Rath, P. D. Markowitz, P. C. Gibbons, W. E. Buhro, Angew. Chem. 2000, 112, 1530-1532; Angew. Chem. Int. Ed. 2000, 39, 1470-1472.

[6] H. Parala, A. Devi, F. Hipler, E. Maile, A. Birkner, H. W. Becker, R. A. Fischer, J. Cryst. Growth 2001, 231, 68-74.

[7] C. H. Liang, L. C. Chen, J. S. Hwang, K. H. Chen, Y. T. Hung, Y. F. Chen, Appl. Phys. Lett. 2002, 81, 22-24.

[8] J. Zhang, L. Zhang, X. Peng, X. Wang, J. Mater. Chem. 2002, 12, $802-804$.

[9] T. Tang, S. Han, W. Jin, X. Liu, C. Li, D. Zhang, C. Zhou, B. Chen, J. Han, M. Meyyappan, J. Mater. Res. 2004, 19, 423-426.

[10] T. V. Shubina, S. V. Ivanov, V. N. Jmerik, D. D. Solnyshkov, V. A. Vekshin, P. S. Kopev, A. Vasson, J. Leymarie, A. Kavokin, H. Amano, K. Shimono, A. Kasic, B. Monemar, Phys. Rev. Lett. 2004, 92, 1174071-1174074.

[11] a) J. Wu, W. Walukiewicz, K. M. Yu, J.W. Ager III, E. E. Haller, H. Lu, W. J. Schaff, Y. Saito, Y. Nanishi, Appl. Phys. Lett. 2002, 80, 3967-3969; b) J. Wu, W. Walukiewicz, S. X. Li, R. Armitage, J. C. Ho, E. R. Weber, E. E. Haller, H. Lu, W. J. Schaff, A. Barcz, R. Jakiela, Appl. Phys. Lett. 2004, 84, 2805-2807.

[12] F. Bechstedt, J. Furthmüller, J. Cryst. Growth 2002, 246, $315-$ 319.

[13] G. Kaczamarczyk, A. Kaschner, S. Reich, A. Hoffmann, C. Thomsen, D. J. As, A. P. Lima, D. Schikora, K. Lischka, R. Averbeck, H. Riechert, Appl. Phys. Lett. 2000, 76, 2122-2124.

[14] A. R. Raju, H. N. Aiyer, C. N. R. Rao, Chem. Mater. 1995, 7, $225-$ 231.

[15] Q. Guo, O. Kato, A. Yoshida, J. Appl. Phys. 1993, 73, 7969971.

[16] A. G. Bhuiyan, A. Hashimoto, A. Yamamoto, J. Appl. Phys. 2003, 94, 2779-2808.

[17] S. T. Barry, D. S. Richeson, Chem. Mater. 1994, 6, 2220-2221.

Received: June 9, 2004

Published online on September 24, 2004 